\title{
UNDERSTANDING THE FEATURES OF INTERNET OF THINGS (IOT) AND Big Data ANalysis
}

\author{
Yew Kee Wong \\ School of Information Engineering, \\ Huang Huai University, Henan, China
}

\begin{abstract}
In the information era, enormous amounts of data have become available on hand to decision makers. Big data refers to datasets that are not only big, but also high in variety and velocity, which makes them difficult to handle using traditional tools and techniques. Due to the rapid growth of such data, solutions need to be studied and provided in order to handle and extract value and knowledge from these datasets. The Internet of Things, or "IoT" for short, is about extending the power of the internet beyond computers and smartphones to a whole range of other things, processes and environments. IoT is at the epicentre of the Digital Transformation Revolution that is changing the shape of business, enterprise and people's lives. This transformation influences everything from how we manage and operate our homes to automating processes across nearly all industries. This paper aims to analyse the relationships of AI, big data and IoT, as well as the opportunities provided by the applications in various operational domains.
\end{abstract}

\section{KEYWORDS}

Artificial Intelligence, Big Data, IoT, Digital Transformation Revolution, Machine Learning.

\section{INTRODUCTION}

When something is connected to the internet, which means that it can send information or receive information, or both. This ability to send and/or receive information makes things smart, and smarter is better. To be smart, a thing doesn't need to have super storage or a supercomputer inside of it. All a thing has to do is connect to super storage or to a supercomputer. Being connected is awesome. Connecting things to the internet yields many amazing benefits. We've all seen these benefits with our smartphones, laptops, and tablets, but this is true for everything else too. And yes, I do mean everything. The Internet of Things (IoT) means taking all the things in the world and connecting them to the internet.

With the development and increase of apps and social media and people and businesses moving online using IoT, there's been a huge increase in data. If we look at only social media platforms, they interest and attract over a million users daily, scaling up data more than ever before. The next question is how exactly is this huge amount of data handled and how is it processed and stored. This is where AI, big data and IoT all 3 components come into play. 


\section{Benefits of Artificial InTELligence}

Artificial intelligence (AI) is a branch of computer science. AI technologies aim to reproduceor surpass abilities in computational systems that are generally deemed intelligent if performed by a human [1]. These abilities include:

- learning

- reasoning

- pattern-recognition

- $\quad$ problem-solving

- $\quad$ visual perception

- language-understanding

\subsection{Different Types of AI}

There are two main types of AI [2]:

Applied AI: is more common and includes systems designed to intelligently carry out a single task, eg move a driverless vehicle, or trade stocks and shares. This category is also known as 'weak' or 'narrow' AI.

Generalised AI: is less common and includes systems or devices that can theoretically handle any task, as they carry enough intelligence to find solutions to unfamiliar problems. Generalised AI is also known as 'strong' AI. Examples of true strong AI don't currently exist, as these technologies are still in very early stages of development.

Many modern AI applications are enabled through a sub-field of AI known as 'machine learning'. What is machine learning? The roots of machine learning (ML) are in statistics. ML uses algorithms and statistical models to perform a specific task without using explicit instructions, instead relying on patterns and inference [3]. For example, ML applications can:

- read a text and decide if the author is making a complaint or a purchase order

- listen to a piece of music and find other tunes to match the mood

- recognise images and classify them according to the elements they contain

- translate large volumes of text in real time

- accurately recognise faces, speech and objects

\subsection{How are AI and Machine Learning used in Business}

Over the years, AI research has enabled many technological advances [4], including:

- $\quad$ virtual agents and chatbots

- $\quad$ suggestive web searches

- $\quad$ targeted advertising

- pattern recognition

- predictive analytics
- voice and speech recognition

- face recognition

- machine translation

- autonomous driving

- automatic scheduling 
Many of these are now commonplace and provide solutions to a great number of business challenges and complex, real-world problems.

\subsection{How are Businesses using AI}

AI is steadily passing into everyday business use. From workflow management to trend predictions, AI has many different uses in business [5]. It also provides new business opportunities.

Application of AI in business:

- Improve customer services - e.g. use virtual assistant programs to provide real-time support to users (for example, with billing and other tasks).

- Automate workloads - e.g. collect and analyse data from smart sensors, or use machine learning (ML) algorithms to categorise work, automatically route service requests, etc.

- Optimise logistics - e.g. use AI-powered image recognition tools to monitor and optimise your infrastructure, plan transport routes, etc.

- Increase manufacturing output and efficiency - e.g. automate production line by integrating industrial robots into your workflow and teaching them to perform labourintensive or mundane tasks [6].

- $\quad$ Prevent outages - e.g. use anomaly detection techniques to identify patterns that are likely to disrupt your business, such as an IT outage. Specific AI software may also help you to detect and deter security intrusions.

- Predict performance - e.g. use AI applications to determine when you might reach performance goals, such as response time to help desk calls.

- Predict behaviour - e.g. use ML algorithms to analyse patterns of online behaviour to, for example, serve tailored product offers, detect credit card fraud or target appropriate adverts.

- Manage and analyse your data - e.g. AI can help you interpret and mine your data more efficiently than ever before and provide meaningful insight into your assets, your brand, staff or customers [7].

- Improve your marketing and advertising - e.g. effectively track user behaviour and automate many routine marketing tasks.

\section{What is Big Data And What Are its Benefits}

Big data analytics has revolutionized the field of IT, enhancing and adding added advantage to organizations. It involves the use of analytics, new age tech like machine learning, mining, statistics and more. Big data can help organizations and teams to perform multiple operations on a single platform, store Tbs of data, pre-process it, analyse all the data, irrespective of thesize and type, and visualize it too [8].

The sources of big data:

\section{Black Box Data}

This is the data generated by air planes, including jets and helicopters. Black box data includes flight crew voices, microphone recordings, and aircraft performance information. 


\section{Social Media Data}

This is data developed by such social media sites as Twitter, Facebook, Instagram, Pinterest, and Google+.

\section{Stock Exchange Data}

This is data from stock exchanges about the share selling and buying decisions made by customers.

\section{Power Grid Data}

This is data from power grids. It holds information on particular nodes, such as usage information.

\section{Transport Data}

This includes possible capacity, vehicle model, availability, and distance covered by a vehicle.

\section{Search Engine Data}

This is one of the most significant sources of big data. Search engines have vast databases where they get their data.

The speed at which data is streamed, nowadays, is unprecedented, making it difficult to deal with it in a timely fashion. Smart metering, sensors, and RFID tags make it necessary to deal with data torrents in almost real-time. Most organizations are finding it difficult to react to data quickly. Not many years ago, having too much data was simply a storage issue [9]. However, with increased storage capacities and reduced storage costs are now focusing on how relevant data can create value.

There is a greater variety of data today than there was a few years ago. Data is broadly classified as structured data (relational data), semi-structured data (data in the form of XML sheets), and unstructured data (media logs and data in the form of PDF, Word, and Text files). Many companies have to grapple with governing, managing, and merging the different data varieties [10].

\subsection{Advantages of Big Data}

1. Today's consumer is very demanding. All customer wants to be treated as an individual and to be thanked after buying a product. With big data, supplier will get actionable data that they can use to engage with their customers one-on-one in real-time [11]. One way bigdata allows supplier to do this is that they will be able to check a complaining customer's profile in real-time and get info on the product(s) the customer is complaining about. Supplier will then be able to perform reputation management.

2. Big data allows supplier to re-develop the products/services they are selling. Information on what others think about their products, such as through unstructured social networking site text helps supplier in product development.

3. Big data allows supplier to test different variations of CAD (computer-aided design) images to determine how minor changes affect their process or product. This makes big data 
invaluable in the manufacturing process.

4. Predictive analysis will keep supplier ahead of their competitors. Big data can facilitate this by, as an example, scanning and analysing social media feeds and newspaper reports. Big data also helps supplier do health-tests on their customers, suppliers, and other stakeholders to help supplier reduce risks such as default.

5. Big data is helpful in keeping data safe. Big data tools help supplier map the datalandscape of their company, which helps in the analysis of internal threats. As an example, supplier will know if their sensitive information has protection or not. A more specific example is that supplier will be able to flag the emailing or storage of 16 digit numbers (which could, potentially, be credit card numbers) [12].

6. Big data allows supplier to diversify their revenue streams. Analysing big data can give supplier trend-data that could help the supplier come up with a completely new revenue stream.

7. The supplier website needs to be dynamic if it is to compete favourably in the crowded online space. Analysis of big data helps supplier personalize the look/content and feel of their site to suit every visitor based on, for example, nationality and sex. An example of this is Amazon's IBCF (item-based collaborative filtering) that drives its "People you may know" and "Frequently bought together" features [13].

8. If the supplier is running a factory, big data is important because the supplier will not have to replace pieces of technology based on the number of months or years they have been in use. This is costly and impractical since different parts wear at different rates. Big data allows supplier to spot failing devices and will predict when the supplier should replace them.

9. Big data is important in the healthcare industry, which is one of the last few industries still stuck with a generalized, conventional approach. Big data allows a cancer patient to get medication that is developed based on his/her genes.

\subsection{Challenges of Big Data}

1. One of the issues with big data is the exponential growth of raw data. The data centres and databases store huge amounts of data, which is still rapidly growing. With the exponential growth of data, organizations often find it difficult to rightly store this data [14].

2. The next challenge is choosing the right big data tool. There are various big data tools, however choosing the wrong one can result in wasted effort, time and money too.

3. Next challenge of big data is securing it. Often organizations are too busy understanding and analysing the data, that they leave the data security for a later stage, and unprotected data ultimately becomes the breeding ground for the hackers.

\section{WHAT IS INTERNET OF THINGS (IOT)}

In the Internet of Things (IoT), all the things can be put into three categories [15]:

1. Sensors that collect information and then send it. 
2. Computers that receive information and then act on it.

3. Things that do both.

And all three of these have enormous benefits that feed on each other:

\section{Collecting and Sending Information}

Sensors can measure temperature, motion, moisture, air quality, light, and almost anything else you can think of. Sensors, when paired with an internet connection, allow us to collect information from the environment which, in turn, helps make better decisions [16]. On a farm, automatically getting information about soil moisture can tell farmers exactly when crops need to be watered. Instead of watering too much or too little (either of which can lead to bad outcomes), the farmer can ensure that crops get exactly the right amount of water. Just as our senses allow us to collect information, sensors allow machines to make sense of their environments [17].

\section{Receiving and Acting on Information}

We're all very familiar with machines acting on input information. A printer receives adocument and then prints it. A garage door receives a wireless signal and the door opens. It's commonplace to remotely command a machine to act. The real power of IoT arises when things can both collect information act on it [18].

\section{Doing Both}

Let's go back to farming. The sensors collect information about the soil moisture. Now, the farmer could activate the irrigation system, or turn it off as appropriate. Instead, the irrigation system can automatically act as needed, based on how much moisture is detected. If the irrigation system receives information about the weather from its internet connection, it can alsoknow when it's going to rain and decide not to water the crops when they'll be watered by the rain any ways [19].

\subsection{How Does IoT Impact You}

The new rule for the future is going to be, "Anything that can be connected, will be connected." But why on earth would a person want so many connected devices talking to each other? There are many examples for what this might look like or what the potential value might be. Say for example you are on your way to a meeting; your car could have access to your calendar and already know the best route to take. If the traffic is heavy your car might send a text to the other party notifying them that you will be late. What if your alarm clock wakes up you at 6 a.m. and then notifies your coffee maker to start brewing coffee for you? What if your office equipment knew when it was running low on supplies and automatically re-ordered more? What if the wearable device you used in the workplace could tell you when and where you were most active and productive and shared that information with other devices that you used while working? Ona broader scale, the IoT can be applied to things like transportation networks: "smart cities" which can help the society to reduce waste and improve efficiency for things such as energy use; this helping the government to understand and improve how everyone work and live [20].

The reality is that the IoT allows for virtually endless opportunities and connections to take place, many of which we can't even think of or fully understand the impact of today. It's not hard to see how and why the IoT is such a hot topic today; it certainly opens the door to a lot of opportunities but also to many challenges. Security is a big issue that is often times brought up [21]. With billions of devices being connected together, what can people do to make sure that their 
information stays secure? Will someone be able to hack into your toaster and thereby get access to your entire network? The IoT also opens up companies all over the world to more security threats. Then we have the issue of privacy and data sharing [22]. This is a hot-button topic even today, so one can only imagine how the conversation and concerns will escalate when we are talking about many billions of devices being connected. Another issue that many companies specifically are going to be faced with is around the massive amounts of data that all of these devices are going to produce. Companies need to figure out a way to store, track, analyse and make sense of the vast amounts of data that will be generated [23].

\section{CONCLuSions}

So this study was concerned by understanding the interrelation between AI, big data and IoT, what frameworks and systems that worked, and how AI can impact the big data analytic process whether by introducing new innovations that foster advanced IoT development process and escalating power consumption, security issues and replacing human in workplaces [24]. The advanced big data analytics and algorithms with various applications show promising results in artificial intelligence development and further evaluation and research using IoT are in progress.

\section{REFERENCES}

[1] Kovach, D., (2017). The Computational Theory of Intelligence: Feedback. InternationalJournal of Modern Nonlinear Theory and Application, 6, pp. 70-73.

[2] J. Wang, (2013). "On the Limit of Machine Intelligence, "International Journal of Intelligence Science, Vol. 3 No. 4, pp. 170-175.

[3] A. Turing, (1950). "Computing Machinery and Intelligence," Mind, Vol. 59, pp. 433-466.

[4] M. Minsky, (1986). "The Society of Mind," Touchstone, Simon \& Schuster, New York, p. 19.

[5] A. Newell and H. Simon, (1958). "Heuristic Problem-Solving: The Next Advance in Operation Research," Operations Research, Vol. 6, No. 6.

[6] A. Clark, (2001). "Mindware: In Introduction to the Philosophy of Cognitive Science," Oxford University Press, New York.

[7] S. Russell and P. Norvig, (2010). "Artificial Intelligence-A Modern Approach," 3rd Edition, Prentice Hall, New Jersey.

[8] S. Del. Rio, V. Lopez, J. M. Bentez and F. Herrera, (2014). On the use of mapreduce for imbalanced big data using random forest, Information Sciences, 285, pp. 112-137.

[9] MH. Kuo, T. Sahama, A. W. Kushniruk, E. M. Borycki and D. K. Grunwell, (2014). Health big data analytics: current perspectives, challenges and potential solutions, International Journal of Big Data Intelligence, 1, pp. 114-126.

[10] R. Nambiar, A. Sethi, R. Bhardwaj and R. Vargheese, (2013). A look at challenges and opportunities of big data analytics in healthcare, IEEE International Conference on Big Data, pp.17-22.

[11] Z. Huang, (1997). A fast clustering algorithm to cluster very large categorical data sets in data mining, SIGMOD Workshop on Research Issues on Data Mining and Knowledge Discovery.

[12] M. D. Assuno, R. N. Calheiros, S. Bianchi, M. a. S. Netto and R. Buyya, (2015). Big data computing and clouds: Trends and future directions, Journal of Parallel and Distributed Computing, 79, pp. 3-15.

[13] I. A. T. Hashem, I. Yaqoob, N. Badrul Anuar, S. Mokhtar, A. Gani and S. Ullah Khan, (2014). The rise of big data on cloud computing: Review and open research issues, Information Systems, 47, pp. 98-115.

[14] L. Wang and J. Shen, (2013). Bioinspired cost-effective access to big data, International Symposium for Next Generation Infrastructure, pp. 1-7.

[15] Mouha, R., (2021). Internet of Things (IoT). Journal of Data Analysis and InformationProcessing, 9, pp. 77-101.

[16] Manyika, et al., (2015). The Internet of Things: Mapping the Value Beyond the Hype. Mckinsey Global Institute, San Francisco.

[17] Bradbury, D., (2015). How Can Privacy Survive in the Era of the Internet of Things? The Guardian, April 7, Sec. Technology.

[18] Gaona-Garcia, P., Montenegro-Marin, C.E., Prieto, J.D., Nieto, Y.V., (2017). Analysis of Security 
Mechanisms Based on Clusters IoT Environments. International Journal of Interactive Multimedia and Artificial Intelligence, 4, pp. 55-60.

[19] Alavi, A.H., Jiao, P., Buttlar, W.G. and Lajnef, N., (2018). Internet of Things-Enabled Smart Cities: State-of-the-Art and Future Trends. Measurement, 129, pp. 589-606.

[20] Zanella, A., Bui, N., Castellani, A., Vangelista, L. and Zorgi, M., (2014). Internet of Thingsfor Smart Cities. IEEE Internet of Things Journal, 1, pp. 22-32.

[21] Khajenasiri, I., Estebsari, A., Verhelst, M. and Gielen, G., (2017). A Review on Internet of Things for Intelligent Energy Control in Buildings for Smart City Applications. Energy Procedia, 111, pp. 770779.

[22] Liu, T., Yuan, R. and Chang, H., (2012). Research on the Internet of Things in the Automotive Industry. ICMeCG 2012, Beijing, pp. 2-13.

[23] Yan, Z., Zhang, P. and Vasilakos, A.V., (2014). A Survey on Trust Management for Internet of Things. Journal of Network and Computer Applications, 42, pp. 120-134.

[24] Palattella, M.R., Dohler, M., Grieco, A., Rizzo, G., Torsner, J., Engel, T. and Ladid, L. , (2016). Internet of Things in the 5G Era: Enablers, Architecture and Business Models. IEEE Journal on Selected Areas in Communications, 34, pp. 510-527.

\begin{abstract}
AUTHOR
Prof. Yew Kee Wong (Eric) is a Professor of Artificial Intelligence (AI) \& Advanced Learning Technology at the HuangHuai University in Henan, China. He obtained his BSc (Hons) undergraduate degree in Computing Systems and a Ph.D. in AI from The Nottingham Trent University in Nottingham, U.K. He was the Senior Programme Director at The University of Hong Kong (HKU) from 2001 to2016. Prior to joining the education sector, he has worked in international technology companies, Hewlett- Packard (HP) and Unisys as an AI consultant. His research interests include AI, online learning, big data analytics, machine learning, Internet ofThings (IOT) and blockchain technology.
\end{abstract}

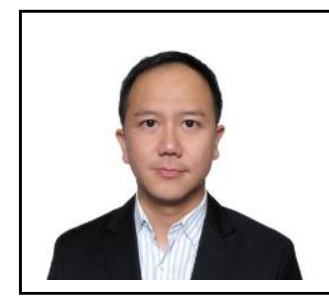

(C) 2021 By AIRCC Publishing Corporation. This article is published under the Creative Commons Attribution (CC BY) license. 